\title{
Peer-to-Peer Multi-Energy and Communication Resource Trading for Interconnected Microgrids
}

Xu, Da; Zhou, Bin; Lu, Nian; Wu, Qiuwei; Voropai, Nikolai; Li , Canbing; Barakhtenko , Evgeny

Published in:

IEEE Transactions on Industrial Informatics

Link to article, DOI:

10.1109/TII.2020.3000906

Publication date:

2021

Document Version

Peer reviewed version

Link back to DTU Orbit

Citation $(A P A)$ :

Xu, D., Zhou, B., Lu, N., Wu, Q., Voropai, N., Li , C., \& Barakhtenko, E. (2021). Peer-to-Peer Multi-Energy and Communication Resource Trading for Interconnected Microgrids. IEEE Transactions on Industrial Informatics, 17(4), 2522 - 2533. https://doi.org/10.1109/TIl.2020.3000906

\section{General rights}

Copyright and moral rights for the publications made accessible in the public portal are retained by the authors and/or other copyright owners and it is a condition of accessing publications that users recognise and abide by the legal requirements associated with these rights.

- Users may download and print one copy of any publication from the public portal for the purpose of private study or research.

- You may not further distribute the material or use it for any profit-making activity or commercial gain

- You may freely distribute the URL identifying the publication in the public portal 


\title{
Peer-to-Peer Multi-Energy and Communication Resource Trading for Interconnected Microgrids
}

\author{
Da Xu, Bin Zhou, Senior Member, IEEE, Nian Liu, Member, IEEE, Qiuwei Wu, Senior Member, IEEE, \\ Nikolai Voropai, Fellow, IEEE, Canbing Li, Senior Member, IEEE, Evgeny Barakhtenko
}

\begin{abstract}
This paper proposes a peer-to-peer (P2P) transactive multi-resource trading framework for multiple multi-energy microgrids. In this framework, the interconnected microgrids not only fulfil the multi-energy demands of with local hybrid biogas-solar-wind renewables, but also proactively trade their available multi-energy and communication resources with each other for delivering secured and high quality of services. The multi-microgrid multi-energy and communication trading is an intractable optimization problem because of their inherent strong couplings of multiple resources and independent decision-makings. The original problem is thus formulated as a Nash bargaining problem and further decomposed into the subsequent social multi-resource allocation subproblem and payoff allocation subproblem. Furthermore, fully-distributed alternating direction method of multipliers (ADMM) approaches with only limited trading information shared are developed to co-optimize the communication and energy flows while taking into account the local resource-autonomy of heterogeneous microgrids. The proposed methodology is implemented and benchmarked on a three-microgrid system over a 24-hourly scheduling periods. Numerical results show the superiority of the proposed scheme in system operational economy and resource utilization, and also demonstrate the effectiveness of the proposed distributed approach.
\end{abstract}

Index Terms-Energy hub, Multi-microgrids, distributed optimization, resource trading, Nash bargaining solution.

\section{INTRODUCTION}

$\mathrm{M}$ icrogrids have been increasingly recognized as a fundamental components of modern energy systems because of their capabilities to accommodate high share of distributed renewable energy sources (RESs) [1]. The inherent intermittency and undispatchability nature of RESs have raised concerns regarding their energy efficiency, economics, security, sustainability, and reliability [2]. Nowadays, in parallel technological development and advancement in smart meters, communication, and management have endowed individuals a more proactive role, and rendered peer-to-peer $(\mathrm{P} 2 \mathrm{P})$ resource trading among microgrids possible [3]. Geographically close microgrids can be cyber-physical interconnected as a multi-microgrid system, where multiple microgrids are collec-

This work was jointly supported by the National Natural Science Foundation of China (51877072), Huxiang Young Talents Programme of Hunan Province under Grant 2019RS2018, and the Open Fund of State Key Laboratory of Alternate Electrical Power System with Renewable Energy Sources. (Corresponding author: Bin Zhou)

D. Xu, B. Zhou, and C. Li are with the College of Electrical and Information Engineering, Hunan University, Changsha 410082, China (e-mail: binzhou@hnu.edu.cn).

N. Liu is with the State Key Laboratory of Alternate Electrical Power System with Renewable Energy Sources, North China Electric Power University, Beijing 102206, China.

Q. Wu is with the Center for Electric Power and Energy, Department of Electrical Engineering, Technical University of Denmark, Kgs. Lyngby, 2800 Denmark.

N. Voropai and E. Barakhtenko are with the Melentiev Energy Systems Institute of Siberian Branch of the Russian Academy of Sciences, Irkutsk 664033, Russia. tively and aggregately managed to coordinate the inherent multi-party energy flow, information flow, and cash flow. Networking multiple autonomous microgrids are thus emerging as strategic efforts for providing multiple high-quality and environmentally friendly energy and information services to local end-users by making full use of limited resources shared by networked microgrids.

So far, extensive literatures on the $\mathrm{P} 2 \mathrm{P}$ resource trading of multiple microgrids can be classified into three types. The first one focuses on the design of leader-follower frameworks for multi-microgrid systems. Leader-follower energy management strategies were studied in [4]-[6], where a microgrid acted as a leader to set incentive signals (price, payment, etc.) for proactive power exchange/trading and other microgrids acted as followers to decide their change/trading profiles in response to the signals. A two-level multiagent-systems based architecture was introduced in [7] to model the power trading behaviors of interconnected microgrids under various market scenarios, and their trading strategies were implemented based on auction algorithms. Auction architectures were applied in [8]-[9] to enable a reserved price based competitive trading among multiple microgrid agents, and their realistic self-interested profit-seeking behaviors were investigated. The second one focuses on the design of aggregation frameworks for multi-microgrid systems. The concept of aggregator was introduced in [10]-[12] to enable the power transactions of microgrids participating the market bidding, and their profit-maximizing rational behaviors under uncertain market environment were analyzed based on the risk management. The fairness and stability of energy sharing among microgrid clusters were investigated in [13] via modeling their self-interests of both buyers and sellers, and an efficient pricing incentive mechanism was developed to encourage the power trading of interconnected microgrids. Virtual aggregators were introduced in [14]-[15] to manage the power sharing for microgrids of prosumers, and a bi-level Stackelberg game was accordingly designed to analyze their rationality and possible responses.

The aforementioned two types of trading frameworks always require a central entity to manage the power exchange/trading flow of multiple agents and also dispatch the cash flow of profits obtained from the energy interactive process. The third type thus aims to design $\mathrm{P} 2 \mathrm{P}$ frameworks for multi-microgrid systems. Various P2P energy-trading platforms were proposed in [16] to encourage the prosumer microgrids to self-organize into coalitions and other community energy initiatives, offering top-down strategies to improve the efficient energy allocations. Different decentralized algorithms have been developed in [17]-[19] for local energy trading in microgrids. Distributed energy sharing frameworks were proposed in [20],[21] to exploit the operational flexibility of combined heat and power unit (CHP) units for P2P thermal and electrical energy trading among multi-microgrids. The concept of consortium blockchain was introduced in [22]-[23] for the secure energy trading, and some technical implementations, 
including credit-based payment scheme and real-time attribution of power losses to each transaction, were studied. Nash bargaining is introduced in [24] to guarantee fair benefit sharing among interconnected microgrids with $\mathrm{P} 2 \mathrm{P}$ power trading, and further extended as a generalized Nash bargaining in [25]. Cooperative game is applied in [26],[27] to model the collectively trading behaviours of microgrids and share the revenue using the Shapley value. Differential game theory and non-cooperative game are applied in [28] to model the strategic interaction of rational microgrid decision-makers for achieving a global coordination. Nevertheless, existing approaches mainly focus on the design of economical mechanisms for $\mathrm{P} 2 \mathrm{P}$ power trading, and few works have attempted to involve the coordinated $\mathrm{P} 2 \mathrm{P}$ trading of multiple resources and technical issues of P2P interconnectivity implementation.

Multi-microgrid systems are typical cyber-physical systems with the implementation of advanced information and communication technologies for wide-area multi-party coordination. Communication resources equipped in these systems include a set of physical computing and communication resource, which offer different cloud-related services to the microgrid operators as well as their end-users [29],[30]. However, in the geo-distributed multi-microgrid system, the communication resources are geographically distributed, and microgrid communication interconnectivity implementation may have several challenges: spatio-temporally distributed bandwidth, limited computing resources, and computation-intensive end-user devices [31]. In addition to the data acquisition from all the microgrid generation, storage, consumption, and communication components, communication links are required among microgrid operators to exchange essential boundary information for global coordination. Rapid emergence and proliferation of advanced smart meters and communication technologies also provide abundant opportunities for proactive end-user demand response programs, which would impose huge computation and communication burdens on the energy-intensive communication devices [29],[30],[32]. Considering such key role of the communication resources in system operation, it is essential to co-optimize the energy and communication resources of microgrids. Reliable communication is inherent in both central, hierarchical, and distributed frameworks for resource-efficient and cost-efficient system operation [2]. The coupling mechanism between the communication flow and power flow is investigated in [31], and a software-defined networking (SDN)-enabled communication network architecture is designed in [33] for microgrid cyber security and resilience enhancement. The integration of SDN and microgrids can be linked to numerous merits among them being: seamless unification of cloud resources, consolidated network management, guaranteed bandwidth, intensified network visibility, and lowered costs of installation/maintenance [33],[34].

Biogas is a promising RES with a wealth of potential to offer significant complementarity to weather-driven solar and wind energy for multi-energy supplies [1]. Biogas is generally produced from local anaerobic digesters, and can be utilized for electricity and heating generations via a CHP unit. The produced raw biogas can be delivered/traded to satisfy multi-energy loads in surrounding/remote areas through pipelines, and also can be further conditioned or upgraded to renewable natural gas pipeline specifications and injected into natural gas network. As an alternative to fossil fuels, the widespread biogas is often highlighted due to its contribution towards formation of cost-competitive and eco-friendly biogas-based renewable microgrid solutions for affordable multi-energy services in the remote areas. Consequently, this paper aims to investigate the $\mathrm{P} 2 \mathrm{P}$ trading of multiple biogas-solar-wind microgrids, including electricity, biogas, and communication resources, so as to leverage their diversified energy/information/cash flows and bring mutual benefits.

Previous works mostly require a central entity to coordinate the energy/information/cash flows in multi-resource trading of multiple microgrids. Actually, the multi-microgrid systems are operated by self-interested entities/managers with heterogeneous profit-seeking preferences, and such centralized operation may bring several disadvantages, such as high bandwidth required for geographic information exchange, inefficient energy management, and long distance to individual operators. It is also envisioned that microgrids will gradually evolve and step into a distributed manner, which can fulfil ever-increasing multi-energy demands of geographically distributed end-user. Various decomposition techniques, including Lagrangian relaxation [20],[21], analytical target cascading [35], alternating direction method of multipliers (ADMM) [24],[25] [36],[37], and consensus algorithm [17],[38] has been successfully applied for decentralized/distributed decision-makings.

This paper proposes a distributed transactive multi-resource trading framework for multiple biogas-solar-wind microgrids. In this framework, each microgrid independently optimizes its allocations of multiple types of resources, and coordinates its generation, storage, consumption, and communication with other microgrids for the exploitation of cyber-physical interconnection. The key contributions of this paper are threefold:

1) A transactive P2P multi-resource framework is proposed for the multi-energy and communication trading of interconnected microgrids. Instead of concerning only the electricity trading in previous works, this paper is devoted to extend the traditional trading model to the $\mathrm{P} 2 \mathrm{P}$ electricity, biogas and communication trading of biogas-solar-wind multi-microgrids. This framework can effectively coordinate internal multi-resource allocations within individual microgrids and external multi-lateral multi-resource trading among networked microgrids to enhance the resource utilization and operational economy.

2) The proactive P2P multi-energy and communication trading process among interconnected microgrids is formulated as a Nash bargaining model to guarantee a fair sharing of trading benefits, and is subsequently decomposed into social multi-resource allocation subproblem and payoff allocation subproblem by leveraging the Nash's axioms.

3) The decomposed P2P multi-resource trading subproblems are further decentralized to the microgrid-based decision-making level and then iteratively solved with the fully-distributed ADMM approaches for optimal synergistic operations of multi-microgrids. The complexity of coordinated P2P trading problem with multiple resources can be reduced, and only a limited amount of trading information exchange is required between adjacent microgrids for privacy protection.

\section{Multi-MiCROGRID SySTEM MODEL}

\section{A. Distributed Multi-Resource Trading Framework}


Fig. 1 illustrates a cluster of $n$ geographically-distributed interconnected biogas-solar-wind microgrids with communication cloudlet resources available. Within each microgrid, wind turbine (WT), photovoltaic thermal (PVT) system, anaerobic digester, and other multi-energy conversion and storage devices, including CHP, furnace, battery energy storage (BES), biogas storage tank, etc., are equipped to condition and convert the locally available biogas-solar-wind renewables into desirable quantities and qualities to fulfil the multi-energy demands. With the deployments of smart meters and advanced metering infrastructures, microgrids are allowed to get access to the electricity market as price-taker and flexible multi-energy loads of end-users are allowed to participate in demand response programs. Cloudlets are a cluster of computing modules and communication modules that are geographically distributed in this multi-microgrid region and managed by the microgrid operators through an SDN framework to provide services to nearby end-users [33]. A cloudlet accomplishes three important tasks [34]: (1) to provide direct computing/communication service as a service provider and cache the required technical data of multi-energy generation, storage, consumption, and communication resources as preparation for the next related service; (2) to manage the communication within and between microgrid operators, such as bridging connections between two nearby operators, collecting demand response applications of end-users, allocating the communication channel, and performing data-sharing service; (3) to serve as an middle layer by offloading resource coordination and supervision requirements to the superior control layer and then return results to the end-users. With the management from the SDN framework as an interlinking layer between the geo-distributed microgrids and communication network, the multi-microgrid system can make full use of the locally available multiple resources and attain complex but efficient resource management functionalities.
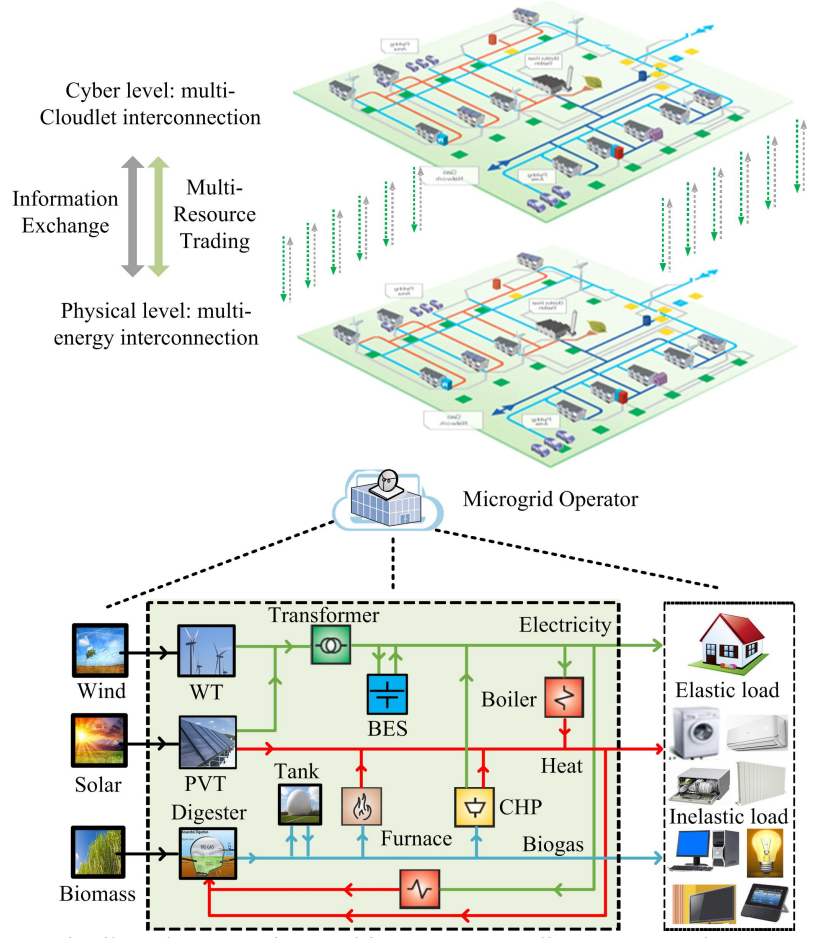

Fig. 1 Distributed Transactive Multi-Resource Trading Framework

Distributed microgrids are physically interconnected using power lines and biogas pipelines, and cyber-interconnected via communication lines. The connected lines/pipelines can be regarded as the edges of networked microgrids and represent geographical proximity, which would compose a P2P resource trading group. Multi-energy and communication resources are increasingly and intensively coupled with each other due to their mutual interactions. For instance, when a microgrid receives many demand response requests during rush hours, its cloudlet could seek resource assistances from surrounding cloudlets since the resource over-utilization may reduce quality of service (QoS) and increase the cloudlet-related energy consumption. It is the aim of this study to co-optimize P2P multi-resource trading behaviors among microgrids to minimize the overall system operating cost. Here, geo-distributed microgrids manage their own supply-demand balance and interact with others through bidirectional communications over $k$ time slots, and resource-rich microgrids are encouraged to trade their locally available resources to the resource-deficient microgrids.

\section{B. Multi-Resource Allocations in Individual Microgrids}

Each microgrid contains the multi-energy generation, storage, consumption, and communication resources.

1) Communication Resources: Since resource over-utilization may reduce QoS, each microgrid operator intends to reduce the communication cost and decrease the overall utilization via resource trading with the surrounding cloudlets. The total communication cost of each microgrid $C_{\mathrm{cr}, k, n}$ in $\$$ involves two parts: cost that microgrid pays for service and penalty of the cloudlet resource over-utilization,

$$
C_{c r, k, n}=\mu_{c r 1} y_{c r, k, n}+\mu_{c r 2}\left(y_{c r, k, n}\right)^{2}
$$

where $\mu_{\mathrm{cr} 1}$ and $\mu_{\mathrm{cr} 2}$ are the unit communication and penalty costs in \$/unit and \$/unit ${ }^{2}$; an upward penalty function $\mu_{\mathrm{cr} 2}(.)^{2}$ in [29] is adopted, and the utilization rate of individual cloudlet $y_{\mathrm{cr}, k, n}$ is evaluatedthroughnormalizingindividualresourcesof communication power $P_{k, n}$ and bandwidth $W_{k, n}$ in a uniformed value [34],

$$
y_{c r, k, n}=\mu_{p} \frac{P_{k, n}}{P_{n, \max }}+\mu_{w} \frac{W_{k, n}}{W_{n, \max }}
$$

where $\mu_{p}$ and $\mu_{w}$ are the user-defined fixed coefficients of two kinds of resources and satisfy the relationship of $\mu_{p}+\mu_{w}=1$; $P_{n, \max }, W_{n, \max }$ are the resource capacities of each cloudlet in $\mathrm{W}$ and $\mathrm{Hz}$.

Assuming that each channel is corrupted by Gaussian white noise [39], the maximum achievable rate $r_{k, n}$, expressed in bit/s, is given by

$$
r_{k, n}=W_{t o t, n} \log _{2}\left(1+\alpha_{k, n} P_{t o t, n} / W_{t o t, n}\right)
$$

where $\alpha_{k, n}$ is the coefficients of bit rate; total communication power and bandwidth $P_{t o t, n}, W_{t o t, n}$ include the individual communication resources $P_{k, n}, W_{k, n}$ and communication resources $T_{p, k, n, n n}, T_{w, k, n, n n}$ traded from other microgrids,

$$
\begin{aligned}
& P_{t o t, k, n}=\sum_{n n \in N \backslash n} T_{p, k, n, n n}+P_{p, k, n} \\
& W_{t o t, k, n}=\sum_{n n \in N \backslash n} T_{w, k, n, n n}+W_{w, k, n}
\end{aligned}
$$

QoS is generally adopted to evaluate the communication services provided to microgrid end-users, and can be estimated as the average operational performance of a cloudlet during a certain time period [32]. To meet the QoS requirement, the time delay experienced by microgrid communication request 
$R_{\mathrm{cr}, k, n}$ should be below a maximum tolerated waiting delay $D_{T, n}$ for a request,

$$
R_{c r, k, n} / r_{k, n} \leq D_{T, n}
$$

where microgrid communication request $R_{\mathrm{cr}, k, n}$ is set as the sum of the normalized elastic electrical and thermal load, since the demand response application requests would be the majority of microgrid communication request under the distributed operational framework.

The power consumption of communication resources includes variable power consumption of information technology (IT) equipment (i.e., computing servers, telecommunication network, etc.) and non-IT constant power consumption (i.e., chillers, fans, base data collection, etc.). Power usage effectiveness (PUE $>1$ ) is defined as the ratio of total power consumption to IT power consumption, which is a famous metric to calculate how much power is used by the computing equipment (in contrast to cooling and other overhead) [32]. Thus, the overall power consumption of communication resources $L_{\mathrm{cr}, k, n}$ in $\mathrm{kW}$ can be estimated as,

$$
L_{c r, k, n}=\left(L_{\text {cridle }}+(P U E-1) L_{c r p e a k}\right)+y_{c r, k, n}\left(L_{c r p e a k}-L_{c r i d l}\right)
$$

where the first term represents the base power usage which does not depend on the resource utilization, and the second term represents the added power usage which indicates the extra power consumption depending on the resource utilization; $L_{\text {cridle }}$ and $L_{\text {crpeak }}$ are the average idle and peak power of communication resources in $\mathrm{kW}$, respectively.

2) Multi-energy Generation: Microgrids can purchase power $P_{\text {buy }, k, n}$ from the electricity market under a time-varying electricity price $\mu_{\text {buy }, k}$ and sell power $P_{\text {sell }, k, n}$ back to distribution system operators under a feed-in tariff contract $\mu_{\text {sell, } k \text { in }}$ $\$ / \mathrm{kWh}$. The electricity procurement cost $C_{\text {grid }, k, n}$ in $\$$ can be formulated as,

$$
C_{\text {grid }, k, n}=\mu_{\text {buy }, k} P_{\text {buy }, k, n}-\mu_{\text {sell }, k} P_{\text {sell }, k, n} \Delta k
$$

Biomass is digested within in a closed thermostatic anaerobic digester with two-layer walls, and its biogas yield $V_{\mathrm{D}, \mathrm{n}}$, measured in $\mathrm{m}^{3}$, can be calculated as,

$$
V_{\mathrm{D}, n}=m T_{\mathrm{Z}, k, n}+b
$$

where $m$ and $b$ are coefficients of biogas production rate; $T_{Z, k, n}$ is the digestion temperature in ${ }^{\circ} \mathrm{C}$ which can be adjusted by controlling the available electricity $S_{\mathrm{ef}, k, n}$ and thermal $S_{\mathrm{hf}, k, n}$ energy for digester heating. The temperature dynamics in the digester can be captured using the following resistor-capacitor based thermodynamics model,

$$
\begin{gathered}
C_{\mathrm{Z}, n} \frac{d T_{\mathrm{Z}, k, n}}{d k}=\eta_{\mathrm{B}} S_{\mathrm{ef}, k, n} \Delta k+S_{\mathrm{hf}, k, n} \Delta k+\frac{T_{\mathrm{W} 1, k, n}-T_{\mathrm{Z}, k, n}}{R_{\mathrm{in}, n}+R_{\mathrm{W} 1, n} / 2} \\
C_{\mathrm{W} 1, n} \frac{d T_{\mathrm{W} 1, k, n}}{d k}=\frac{T_{\mathrm{Z}, k, n}-T_{\mathrm{W} 1, k, n}}{R_{\mathrm{in}, n}+R_{\mathrm{W} 1, n} / 2}+\frac{T_{\mathrm{W} 2, k, n}-T_{\mathrm{W} 1, k, n}}{R_{\mathrm{W} 2, n} / 2+R_{\mathrm{W} 1, n} / 2} \\
C_{\mathrm{W} 2, n} \frac{d T_{\mathrm{W} 2, k, n}}{d k}=\frac{T_{\text {out }, k, n}-T_{\mathrm{W} 2, k, n}}{R_{\text {out }, n}+R_{\mathrm{W} 2, n} / 2}+\frac{T_{\mathrm{W} 1, k, n}-T_{\mathrm{W} 2, k, n}}{R_{\mathrm{W} 1, n} / 2+R_{\mathrm{W} 2, n} / 2} \\
T_{\mathrm{Z}, \text { min }} \leq T_{\mathrm{Z}, k, n} \leq T_{\mathrm{Z}, \text { max }}
\end{gathered}
$$

where $T_{o u t, k, n}, T_{Z, k, n}, T_{W 1, k, n}$ and $T_{W 2, k, n}$ are the temperatures of digester outside, inside, the $1^{\text {st }}$ and $2^{\text {nd }}$ layer walls in ${ }^{\circ} \mathrm{C}$; their external, internal convective heat transfer, conductive heat transfer of the two-layer walls are calculated based on the material thermal resistance $R_{o u t, n}, R_{i n, n}, R_{W 1, n}, R_{W 2, n}$ in ${ }^{\circ} \mathrm{C} / \mathrm{kW}$ and material thermal capacitances $C_{Z, n}, C_{W 1, n}, C_{W 2, n}$ in $\mathrm{kWh} /{ }^{\circ} \mathrm{C} ; \eta_{\mathrm{B}}$ is the electrical-thermal efficiency of boiler; $T_{Z, \max }$ and $T_{Z, \text { min }}$ are the upper and lower values of digestion temperature to guarantee the survival of anaerobic organisms in ${ }^{\circ} \mathrm{C}$, respectively. A linearization method for generic nonlinear differential systems in [40] is then applied to linearize the thermo-electrochemical dynamics in (2b)-(2e) around the nearest equilibrium point of the system operating state. It has been demonstrated in [1],[40] that this linearization would not lead to significant truncation errors as a consequence of the small digestion temperature range.

The outputs of boiler $S_{\mathrm{B}, k, n}$, furnace $S_{\mathrm{F}, k, n}$, and CHP $S_{\mathrm{CHP}, k, n}$ should be subject to their capacity limits $S_{\mathrm{B}, n, \max }, S_{\mathrm{F}, n, \max }$, $S_{\mathrm{CHP}, n, \max }$ in $\mathrm{kW}$,

$$
0 \leq S_{i, k, n} \leq S_{i, n \text {,max }} \quad i=\mathrm{CHP}, \mathrm{B}, \mathrm{F}
$$

3) Multi-energy Consumption: The multi-energy loads of each microgrid include three types: electricity load $L_{e, k, n}$ in $\mathrm{kW}$, heat/thermal load $L_{h, k, n}$ in $\mathrm{kW}$, and gas load $L_{g, k, n}$ in $\mathrm{m}^{3}$. Each type of load can be classified into three parts: inelastic/base loads $L_{\text {ebase }, k, n}$, Lhbase,k,n (e.g., lighting and refrigerator which could not shifted), elastic loads $L_{e i n, k, n}$, Lhin,k,n (e.g., dish-washer and water heater which could be shifted within a day), and communication energy consumption $L_{c r, k, n}$. The microgrids can implement the demand response by coordinating the elastic loads for ensuring the reliable and secure operation [24],[37], which would incur the discomfort $\operatorname{cost} C_{\text {load, }, k, n}$ in $\$$ of by deviation from their preferred load demand level $L_{e p r e, k, n}, L_{h p r e, k, n}$,

$$
\begin{gathered}
C_{\text {load }, k, n}=\mu_{\text {load }}\left[\left(L_{e i n, k, n}-L_{\text {epre }, k, n}\right)^{2}+\left(L_{h i n, k, n}-L_{h p r e, k, n}\right)^{2}\right] \\
L_{e, k, n}=L_{e b a s e, k, n}+L_{c r, k, n}+L_{e i n, k, n} \\
L_{h, k, n}=L_{h b a s e, k, n}+L_{h i n, k, n}
\end{gathered}
$$

where $\mu$ load is unit discomfort cost for load deviation in $\$ / \mathrm{kW}^{2}$; the scheduled elastic loads during the demand response program should satisfy the following constraints:

$$
\begin{aligned}
& \sum_{k \in K} L_{e i n, k, n}=D_{e i n, n} \\
& \sum_{k \in K} L_{h i n, k, n}=D_{h i n, n} \\
& 0 \leq L_{e i n, k, n} \leq L_{e i n, \text { max }, k, n} \\
& 0 \leq L_{h i n, k, n} \leq L_{h i n, \text { max }, k, n}
\end{aligned}
$$

where $D_{e i n, n}, D_{h i n, n}$ correspond to the total elastic loads in the entire operation horizon in $\mathrm{kW}$. $L_{e i n, \max , k, n}, L_{h i n, \max , k, n}$, measured in $\mathrm{kW}$, provide a upper bound for the elastic energy consumption of microgrid.

4) Multi-energy Storage: Energy storages, including BESs and biogas tanks, are equipped in each microgrid, which can store the intermittent renewable power generations as electricity and biogas and make profits from the sale of electricity. Frequent charging $P_{\mathrm{ch}, k, n}$ and discharging $P_{\mathrm{dis}, k, n}$ of BES in $\mathrm{kW}$ would cause a certain degree of wear and tear, thereby degrading its cycling numbers and capacity. The battery degradation cost $C_{\mathrm{BES}, k, n}$ in $\$$ can be formulated as,

$$
C_{\mathrm{BES}, k, n}=\mu_{\mathrm{BES}}\left(P_{\mathrm{ch}, k, n}+P_{\mathrm{dis}, k, n}\right) \Delta k
$$

where $\mu_{\mathrm{BES}}$ is the unit average/amortized degradation cost of charging/discharging over the whole service time in $\$ / \mathrm{kWh}$ which can be calculated with its capital cost, cycling numbers, capacity, and reference state of charge (SOC) according to [21]. Constraints (4a)-(4f) limit the SOC and charging/discharging of BES and biogas tank, 


$$
\begin{gathered}
S O C_{\mathrm{BES}, k, n}=S O C_{\mathrm{BES}, k-1, n}+\frac{\eta_{\mathrm{ch}} P_{\mathrm{ch}, k-1, n} \Delta k}{E_{\mathrm{R}, n}}-\frac{P_{\mathrm{dis}, k-1, n} \Delta k}{\eta_{\mathrm{dis}} E_{\mathrm{R}, n}} \\
S O C_{j, \text { min }} \leq S O C_{j, k, n} \leq S O C_{j, \max }(j=\mathrm{BES}, \mathrm{bio}) \\
0 \leq P_{\mathrm{ch}, k, n} \leq P_{\mathrm{ch}, n, \text { max }} \\
0 \leq P_{\mathrm{dis}, k, n} \leq P_{\mathrm{dis}, n, \text { max }} \\
S O C_{\mathrm{bio}, k, w}=S O C_{\mathrm{bio}, k-1, w}-V_{\mathrm{GS}, k-1, w} / V_{\mathrm{R}, w} \\
V_{\mathrm{GS}, n, \min } \leq V_{\mathrm{GS}, k, n} \leq V_{\mathrm{GS}, n, \text { max }}
\end{gathered}
$$

where $\mathrm{E}_{\mathrm{R}, \mathrm{n}}$ is the BES capacity in $\mathrm{kWh} ; S O C_{j, k, n}$ is the current SOC; $\eta_{\mathrm{ch}}$ and $\eta_{\text {dis }}$ are BES charging and discharging efficiencies; $S O C_{j, \max }$ and $S O C_{j, \text { min }}$ are the maximum and minimum values of SOC; $P_{\mathrm{ch}, \max }$ and $P_{\mathrm{dis}, \max }$ are charging and discharging limits in $\mathrm{kW} ; V_{\mathrm{R}, \mathrm{n}}$ is the volume of biogas storage in $\mathrm{m}^{3}$; $V_{\mathrm{GS}, k}$ is the net output of biogas storage (discharging minus charging) in $\mathrm{m}^{3} ; V_{\mathrm{GS}, \max }$ and $V_{\mathrm{GS}, \min }$ are the maximum and minimum values of storage output in $\mathrm{m}^{3}$.

\section{P2P Multi-Resource Trading among Microgrids}

Resource trading is essential for microgrids to improve the resource utilization and operational economy. Geo-distributed microgrids may have different RESs and local multi-energy loads due to their energy preferences and weather-dependent nature. The produced biogas and electricity are versatile and flexible energy resources, which can either traded to other microgrids via power lines and biogas pipelines, or be directly satisfy the local load demands of individual microgrid via multi-energy converters and storages. In addition to multi-energy complementarity of biogas-solar-wind renewable portfolio, demand response programs would be implemented to maintain a global supply-demand balance with the locally available and traded communication resources. Here, each microgrid could negotiate with other networked microgrids about the amounts of resource trading $T_{a, k, n, n n}$ and the associated payment $C_{a, k, n, n n}$. Noted that electricity, biogas, communication power and bandwidth are four types of trading resources, and specified as "e", "g", " $p ", " w$ ". The multi-resource trading and corresponding payment among microgrids should be subjected to the following market clearing constraints,

$$
\begin{array}{ll}
T_{\mathrm{a}, k, n, n n}+T_{\mathrm{a}, k, n n, n}=0 & a=e, g, p, w \\
C_{\mathrm{a}, n, n n}+C_{\mathrm{a}, n n, n}=0 & a=e, g, p, w
\end{array}
$$

where $T_{a, k, n, n n}$ is the amount of resource $a$ that microgrid $n$ exchanges with microgrid $n n$, and $C_{a, k, n, n n}$ is the associated payment for trading of resource $a$ between microgrid $n$ and $n n$.

Each microgrid operator coordinates its communication resources (1)-(1f), multi-energy generations (2)-(2f), consumptions (3)-(3f), storages (4)-(4f), and tradings (5a)-(5b) to ensure the multi-energy supplies for the consumers,

$$
\begin{aligned}
& L_{e, k, n}= P_{\mathrm{WT}, k, n}+P_{\mathrm{PVT}, k, n}+P_{\mathrm{dis}, k, n}-P_{\mathrm{ch}, k, n}-S_{\mathrm{B}, k, n} / \eta_{\mathrm{B}} \\
&+ S_{\mathrm{CHP}, k, n}-S_{\mathrm{ef}, k, n}-\sum_{n n \in N \backslash n} T_{\mathrm{e}, k, n, n n}+P_{\mathrm{buy}, k, n}-P_{\mathrm{selll}, k, n} \\
& L_{h, k, n}=H_{\mathrm{PVT}, k, n}+S_{\mathrm{B}, k, n}+S_{\mathrm{CHP}, k, n} \eta_{h, \mathrm{CHP}} / \eta_{e, \mathrm{CHP}}+S_{\mathrm{F}, k, n}-S_{\mathrm{hf}, k, n} \\
& L_{g, k, n}=V_{\mathrm{D}, k, n}+V_{\mathrm{GS}, k, n}-S_{\mathrm{CHP}, k, n} \Delta k / Q_{\mathrm{bio}} \eta_{e, \mathrm{CHP}} \\
&-S_{\mathrm{F}, k, n} \Delta k / Q_{\mathrm{bio}} \eta_{\mathrm{F}}-\sum_{n n \in N \backslash n} T_{\mathrm{g}, k, n, n n}
\end{aligned}
$$

where $P_{\mathrm{WT}}, P_{\mathrm{PVT}}, H_{\mathrm{PVT}}$ are outputs of $\mathrm{WT}$ and PVT in $\mathrm{kW}$; $\eta_{\mathrm{e}, \mathrm{CHP},} \eta_{\mathrm{h}, \mathrm{CHP}}$, and $\eta_{\mathrm{F}}$ are electrical and thermal efficiencies of CHP and furnace; $Q_{\text {bio }}$ is heating value of biogas in $\mathrm{kWh} / \mathrm{m}^{3}$.

Each microgrid operator aims to minimize its total operating cost $C_{\mathrm{non}, n}$ in $\$$, consisting of the communication $\operatorname{cost} C_{\mathrm{cr}, k, n}$, electricity procurement $\operatorname{cost} C_{\text {grid, }, k, n}$, users' discomfort costs $C_{\text {load }, k, n}$, and battery degradation $\operatorname{cost} C_{\mathrm{BES}, k, n}$,

$$
C_{\text {non }, n}=\sum_{k \in K}\left(C_{\mathrm{BES}, k, n}+C_{\mathrm{load}, k, n}+C_{\mathrm{grid}, k, n}+C_{\mathrm{cr}, k, n}\right)
$$

\section{Proposed Solution Methodology}

The multi-microgrid P2P multi-energy and communication trading (1)-(7) cannot be easily solved using standard commercial solvers due to the conflict of interests, couplings of multi-energy supply-demand, resource trading, and payments. The coordinated P2P multi-resource trading of numerous microgrids requires multi-party operating states and technical parameters. However, it is not realistic that all the geo-distributed microgrids are governed by a single entity/manager and all the information are unconditionally shared with each other. Thus, a game-theoretic transactive bargaining framework is firstly designed to facilitate a mutually beneficial multi-resource trading outcomes among microgrids through negotiation and coordination. Then, the formulated multi-resource trading problem is decentralized into multiple microgrid-level decision-making subproblems and iteratively solved using the fully-distributed ADMM approaches with limited information shared.

\section{A. Bargaining based Transactive Multi-resource Trading}

In the multi-microgrid bargaining problem, the self-interested microgrid operators negotiate with each other to achieve a mutually beneficial agreement, which are better for both players than the disagreement point. Various bargaining solutions [41], including Kalai-Smorodinsky bargaining solution, Egalitarian bargaining solution, and Nash bargaining solution, have been developed by assuming slightly different bargaining properties of the final agreement point. These bargaining solutions are obtained through maximizing the product of additional benefit, equalizing the ratios of maximal gains, and maximizing the minimum payoff. It has been systematically proved in [41] that Nash bargaining theory provides a fair Pareto optimal solution that satisfies the four axioms of symmetry, Pareto optimality, scale invariance, and independence of irrelevant alternatives. Mathematically, taking two-player bargaining problem as example, a pair of payoffs $\left(u_{1}, u_{2}\right)$ is a Nash bargaining solution only if it solves the following optimization problem:

$$
\begin{aligned}
& \max _{u_{1}, u_{2}}\left(u_{1}-d_{1}\right)\left(u_{2}-d_{2}\right) \\
& \text { s.t. }\left(u_{1}, u_{2}\right) \in U ;\left(u_{1}, u_{2}\right)>\left(d_{1}, d_{2}\right)
\end{aligned}
$$

where $U$ is a convex feasibility set and its elements can be interpreted as agreements; $d_{1}$ and $d_{2}$ are payoffs of two players from the disagreement point; the product of the two excess benefits $\left(u_{1}-d_{1}\right)\left(u_{2}-d_{2}\right)$ is generally referred to as the Nash product.

In this work, the multi-lateral resource trading negotiation problem is modelled and analyzed using the Nash bargaining theory [42]. Each microgrid bargains with other microgrids for the amount of resources and corresponding payment. The extra payment $\operatorname{cost} C_{\mathrm{T}, n}$ in $\$$ to other microgrids can be expressed as follows, 


$$
C_{\mathrm{T}, n}=\sum_{a} \sum_{n n \in N \backslash n} C_{\mathrm{a}, n, n n}
$$

The overall cost of microgrid $n C_{\mathrm{tot}, n}$ in $\$$ can be expressed as,

$$
C_{\mathrm{tot}, n}=\sum_{k \in K}\left(C_{\mathrm{BES}, k, n}+C_{\text {load }, k, n}+C_{\mathrm{grid}, k, n}+C_{\mathrm{cr}, k, n}\right)+C_{\mathrm{T}, n}
$$

All microgrid operators are rational and expected to enhance their resource utilization through implementing the cost-minimization resource trading. Certainly, microgrids will not cooperate if the bargaining process cannot reduce their cost,

$$
C_{\mathrm{tot}, n} \leq C_{\mathrm{wt}, n}^{*}
$$

where $C_{\mathrm{wt}, n}^{*}$ is the so-called "disagreement point" which is the minimum of the $C_{\text {non, } n}$ that microgrid $n$ can obtain without trading resources with other microgrids.

The mathematical formulation of our proposed Nash bargaining based multi-resource trading problem is given as,

$$
\begin{aligned}
& \max \prod_{n \in N}\left(C_{\mathrm{wt}, n}^{*}-C_{\mathrm{tot}, n}\right) \\
& \text { s.t. }(1)-(10)
\end{aligned}
$$

By leveraging the Nash's axioms, the formulated multi-resource trading problem (11) can be equivalently decomposed as two subproblems: social multi-resource allocation subproblem (12) and payoff allocation subproblem (13),

$$
\begin{aligned}
& \min \sum_{n \in N} C_{\mathrm{non}, n} \\
& \text { s.t. }(1)-(4 \mathrm{f}),(5 \mathrm{a}),(6 \mathrm{a})-(9) \\
& \max \prod_{n \in N}\left(C_{\mathrm{w}, n}^{*}-C_{\mathrm{non}, n}^{*}-C_{\mathrm{T}, n}\right) \\
& \text { s.t. }(5 \mathrm{~b}),(10)
\end{aligned}
$$

where $C_{\text {non }, n}^{*}$ is the optimal value of the subproblem (12).

Remark 1: Due to the Pareto efficiency and convexity properties of this problem [41]-[43], both existence and uniqueness for Nash bargaining solution of problem (11) can be guaranteed. It also can be found that the involved decision variables of the Nash bargaining problem (11) can be divided into two decoupled sets: the energy/communication allocation variables and trading payment variables. Given the optimal multi-energy and communication allocation decisions, the optimal trading payment decisions from (13) can be obtained as,

$$
C_{\mathrm{T}, n}=C_{\mathrm{w}, n}^{*}-C_{\mathrm{non}, n}^{*}-\sum_{n \in N}\left(C_{\mathrm{w}, n}^{*}-C_{\mathrm{non}, n}^{*}\right) / N
$$

Substitute (14) into problem (11) and yield the optimal objective:

$$
\left[\sum_{n \in N}\left(C_{\mathrm{wt}, n}^{*}-C_{\mathrm{non}, n}^{*}\right) / N\right]^{N}
$$

It thus can be proved that problem (11) minimizes the social cost of all microgrids in subproblem (12), and optimal multi-resource trading solutions can be obtained by sequentially solving subproblem (12) and (13).

\section{B. Distributed Algorithms}

Here, the couplings of the subproblem (12) and (13) is the resource trading and payment constraints $(5 a)-(5 b)$, which can be further decomposed as a set of microgrid subproblems using the ADMM approaches to protect the information privacy and local decision-making autonomy. Self-interested microgrids rationally behave as profit-maximizing and share necessary trading information with its surroundings. Algorithm 1 shows the detailed procedure of the distributed approaches. Due to their convexity properties of (12) and (13), the convergence of Algorithm 1 can always be guaranteed and the algorithm would gradually converge to the optimal value of the problem within finite iterations. Details of the proof are given in [43].

Algorithm 1 Distributed algorithm solving (12) and (13)

1: Input multi-energy generation, storage, consumption, and communication parameters of multi-microgrid system.

2: Set iteration index $i t=0$, tolerances $\delta_{1}, \delta_{2}$. Initialize Lagrangian multipliers $\mathbf{y}, \mathbf{c y}$, step sizes $\mathbf{d}$, $\mathbf{c d}$.

3: Each microgrid parallelly solves the social multi-energy and communication allocation subproblem with local constraints (1)-(4),(6)-(9) and the following Lagrangian function of objective (12):

$$
\begin{aligned}
\mathbf{T}_{\mathrm{a}, k, n, n n}^{i t+1} & =\arg \min \left\{C_{\mathrm{non}, n}+\frac{\mathbf{d}_{\mathrm{a}, n, n n}}{2} \sum_{a} \sum_{k \in K} \sum_{n n \in N \backslash n} \| \mathbf{T}_{\mathrm{a}, k, n, n n}\right. \\
& \left.-\mathbf{T}_{\mathrm{a}, k, n, n n}^{i t}+\mathbf{T}_{\mathrm{a}, k, n, n, n n}^{i t, n e r}+\mathbf{y}_{\mathrm{a}, k, n, n n}^{i t} \|_{2}^{2}\right\}
\end{aligned}
$$

where superscript aver represents the average.

4: Calculate and check if residual is less than the predefined tolerances:

$$
\max \sum_{k \in K} \sum_{n n \in N / n}\left\|\mathbf{T}_{\mathrm{a}, k, n, n n}^{i+1+1}+\mathbf{T}_{\mathrm{a}, k, n n, n}^{i+1}\right\|_{2}^{2} \leq \delta_{1}
$$

Once satisfied, iteration ends. Otherwise, each microgrid updates its $\mathbf{y}$ :

$$
\mathbf{y}_{\mathrm{a}, k, n, n n}^{i t+1}=\mathbf{y}_{\mathrm{a}, k, n, n n}^{i t}+\mathbf{T}_{\mathrm{a}, k, n, n n}^{i t+1, a v e r}
$$

5: Set $i t=i t+1$. Each microgrid repeats Steps 3-4 until the stopping criteria are satisfied.

6: Each microgrid parallelly solves the payment bargaining subproblem with local constraints (10) and the following Lagrangian function of objective (13):

$$
\begin{aligned}
\mathbf{C}_{\mathrm{a}, n, n n}^{i t+1}= & \arg \min \left\{-\ln \left(C_{\mathrm{w}, n}^{*}-C_{\mathrm{non}, n}^{*}-C_{\mathrm{T}, n}\right)+\frac{\mathbf{c d}_{\mathrm{a}, n, n n}}{2}\right\} \\
& \left.\sum_{a} \sum_{n n \in N \backslash n}\left\|\mathbf{C}_{\mathrm{a}, n, n n}-\mathbf{C}_{\mathrm{a}, k, n, n n}^{i t}+\mathbf{C}_{\mathrm{a}, k, n, n n}^{i t, a v r}+\mathbf{c y}_{\mathrm{a}, k, n, n n}^{i t}\right\|_{2}^{2}\right\}
\end{aligned}
$$

7: Calculate and check if residual is less than the predefined tolerances:

$$
\max \sum_{n n \in N / n}\left\|\mathbf{C}_{\mathrm{a}, n, n n}^{i t+1}+\mathbf{C}_{\mathrm{a}, n n, n}^{i t+1}\right\|_{2}^{2} \leq \delta_{2}
$$

Once satisfied, iteration ends. Otherwise, each microgrid updates its cy:

$$
\mathbf{c y}_{\mathrm{a}, n, n n}^{i t+1}=\mathbf{c y}_{\mathrm{a}, n, n n}^{i t}+\mathbf{C}_{\mathrm{a}, n, n n}^{i t+1, a v e r}
$$

8: Set $i t=i t+1$. Each microgrid repeats Steps 6-7 until the stopping criteria are satisfied.

\section{CAse Studies}

\section{A. System Description}

The proposed distributed transactive multi-energy and communication trading methodology is benchmarked on a three-microgrid system in Hunan, China. The schematic diagram of the studied multi-microgrid system is given in Fig.1. The retailed electricity price is obtained from [24], and the feed-in price is set as $0.05 \$ / \mathrm{kWh}$. The data profiles of multi-energy generations, conversions, storages, and base energy consumptions of three microgrids in this study are obtained from [1],[21],[24],[37]. Here, $L_{\text {cridle }}$ and $L_{\text {crpeak }}$ are set as $5 \mathrm{~kW}$ and $15 \mathrm{~kW}$; $L_{e p r e, k, n}, L_{h p r e, k, n}, D_{e i n, n}, D_{h i n, n}$ of each microgrid are 
all set as $30 \%$ of each type of base load, and $L_{e i n, \max , k, n}$, $L_{h i n, \max , k, n}$ are set as $60 \%$ of each type of load at the $k$ th time slot [44]. Other parameters from [29]-[30][32],[34],[39] in per unit values are summarized as follows: $\mathrm{PUE}=1.2, \mu_{\mathrm{cr} 1, \mathrm{n}}=[1,5$, 10], $\mu_{\mathrm{cr} 2 \mathrm{n}}=[1,5,10], D_{T, n}=[2,2.4,2.8], \alpha_{k, n}=[2,2.4,2.8]$, $P_{n, \max }=[0.5,1,2], W_{n, \max }=[0.5,1,2], \mu_{p}=\mu_{w}=0.5$. The tolerances for the distributed algorithm are $10^{-5}$ and the step size is 1. The multi-energy and communication trading are implemented over a 24-hourly scheduling periods, and all the simulations are performed and coded using the commercial platform GAMS [45] on a laptop with 2.3-GHz Intel Core i5 CPU and 8GB RAM. The centralized and distributed problems are solved using the NLP solver CONOPT with their default settings.

\section{B. Comparative Results and Analysis}

Three comparative schemes are implemented: 1) Scheme 1 is the proposed distributed transactive multi-resource trading scheme in Sections II-IV; 2) Scheme 2 is the multi-microgrid multi-resource trading without taking into account the external communication trading; 3) Scheme 3 performs the multi-microgrid scheduling without taking into account external multi-energy and communication trading.

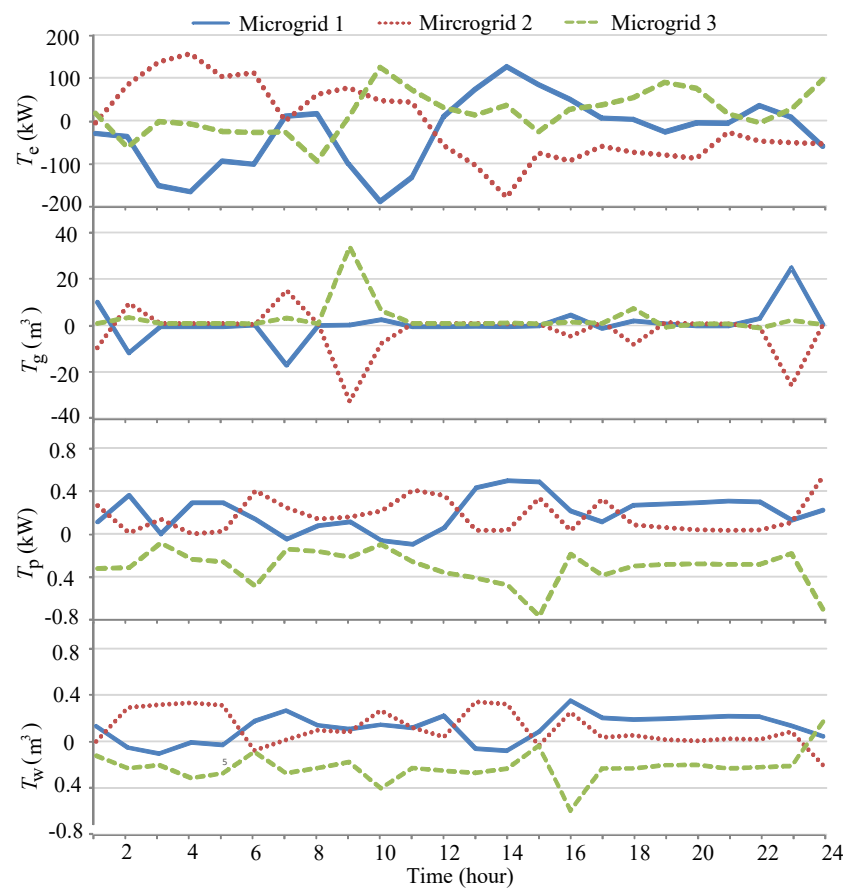

Fig. 2 Power, biogas, and communication trading among microgrids in scheme 1

Fig. 2-3 illustrate the curves of daily electricity, biogas, communication trading among microgrids in schemes 1-2. Fig. 4-5 illustrate electricity procurement and resource utilization of microgrids in schemes 1-3. It can be found from Fig. 2-3 that all three microgrids interactively trade energy and communication resources with each other across the 24-hour operation horizon. During hours 1-12, microgrids 1 and 3 have relatively higher available renewable outputs than microgrid 2 , thus sell excessive energy to microgrid 2. Due to the sudden drop of renewable power supply, microgrids 1 and 3 have to purchase energy from microgrid 2 in hours 11-24. As for biogas, as a consequence of larger thermal load and gas load, the locally available biogas is delivered from microgrid 2 to mi- crogrid 3 and 1 during hours $8-10$ and hours 22-24. Though energy trading among microgrids incur extra payments, it enables the renewable accommodation during hours 2-5 instead of feeding back to the grid. Thus, less electricity is purchased by microgrid 1 from the market than other scheme, decreasing their operating costs, as shown in Fig.4. On the other hand, the multi-microgrid system can improve its communication capability by trading resource from surrounding microgrids in the case of resource shortage. Due to the larger communication requirements for electrical and thermal demand response, resource-rich microgrid 3 shares its communication resource to resource-deficient microgrids 1 and 2 in addition to meeting the internal requirements. However, in the scheme 2 and 3 without communication sharing, the microgrid 1 always stays at a high communication resource utilization. Through offloading request on the surrounding cloudlets with low occupation, the average resource utilization of microgrid 1 and 2 are significantly reduced, as shown in Fig.5.

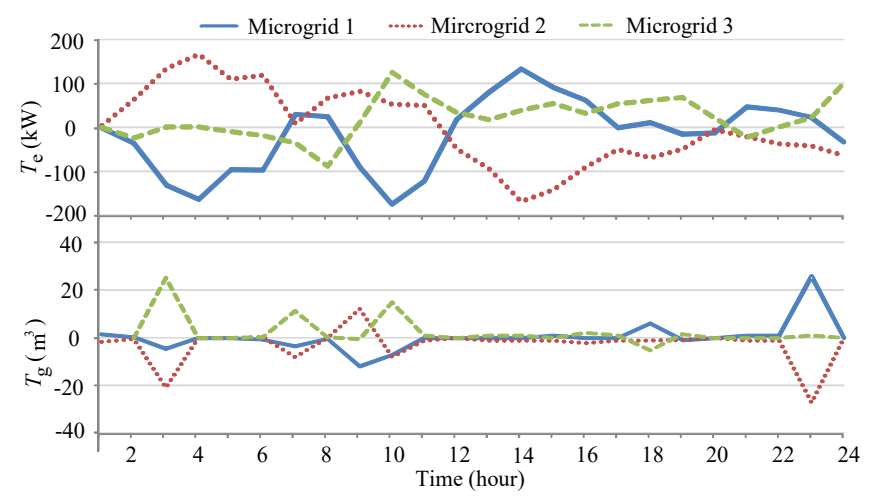

Fig. 3 Power and biogas trading among microgrids in scheme 2

Fig.6 shows the optimized electrical load curve of microgrids 1-3 in scheme 1. In such multi-microgrid system, renewable energy, load curve, and electricity price have significantly different peak periods. Due to different types of consumers, original load curve of microgrids $1-3$ reach their peak values during 13-22, 11-14, and 10-18. While there are abundant renewable generations during hours 1-10 (wind energy) and hour 11-15 (solar energy), the electricity price is much higher during on-peak hours 11-20. It can be found that all microgrids shift their elastic loads from on-peak periods to off-peak time periods. For example, more renewable energy is consumed during morning hours and less electricity is purchased during on-peak hours.

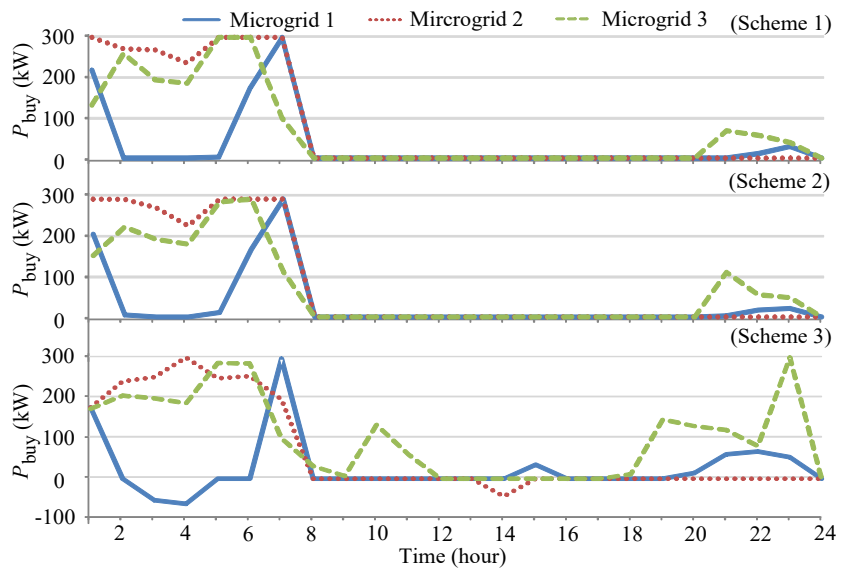

Fig. 4 Gird power procurement of microgrids in schemes 1-3 


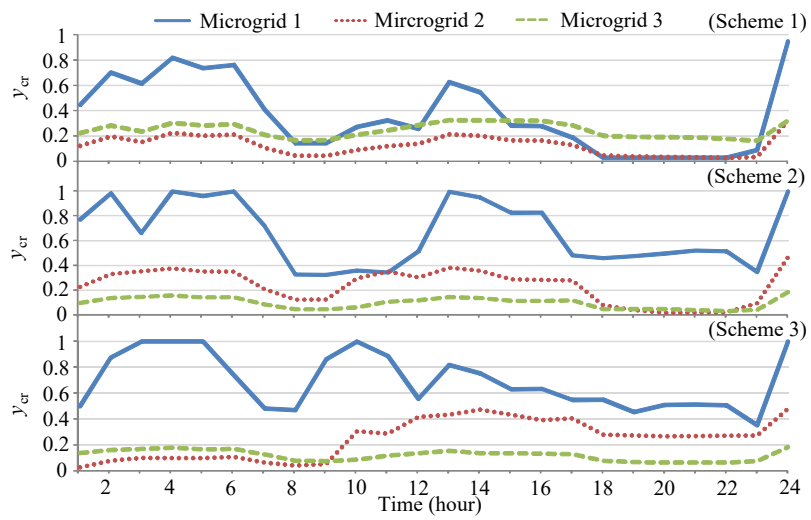

Fig. 5 Resource utilization of cloudlets in schemes 1-3

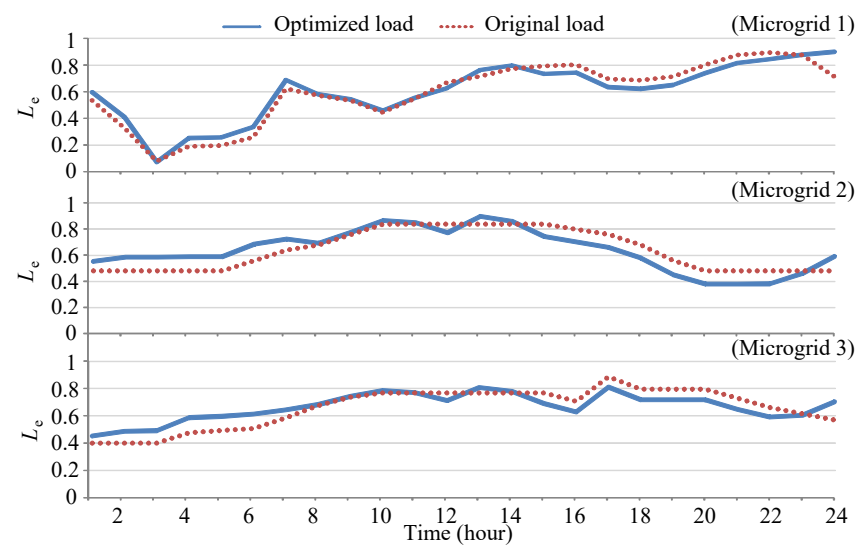

Fig. 6 Demand response of microgrids in schemes 1-3

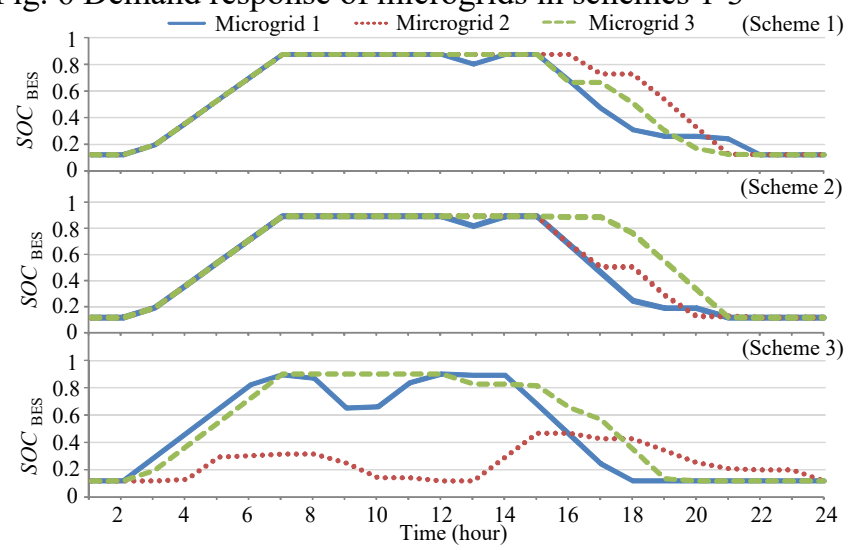

Fig. 7 SOC of BES of microgrids in schemes 1-3

Fig. 7-9 shows the outputs of multi-energy converters and storages of three microgrids with schemes 1-3. It can be seen that compared with schemes without multi-resource trading, the proposed methodology can attain better synergies of inner multi-resource scheduling and outer multi-resource trading. More specifically, during the morning and noon hours, abundant renewable generations serve as the mainly energy sources for multi-energy supplies and BES charging, while CHP and furnace stays unchanged. During the hours 17-24 when there is a dramatical decrease of renewable generations, the BES and CHP in scheme 1 sharply increase to fulfil the rising multi-energy demands while the furnace and boiler remains unchanged. With the multiple traded resources from each other, the outputs of biogas storage and high-efficiency CHP in scheme 1 stay at a high level to fulfil the on-peak multi-energy loads during the hours 21-24. However, in scheme 3, the biogas storage and furnace increase their outputs to follow the heating load while CHP decreases its output. Batteries are also not fully charged with an additional reverse discharging during hours 8-11 and exhausted in advance. Furthermore, the CHP is prioritized as the multi-energy generation plants during hours 17-24 because of its higher conversion efficiency, while the remaining part between CHP outputs and multi-energy load are satisfied with boiler and furnace.

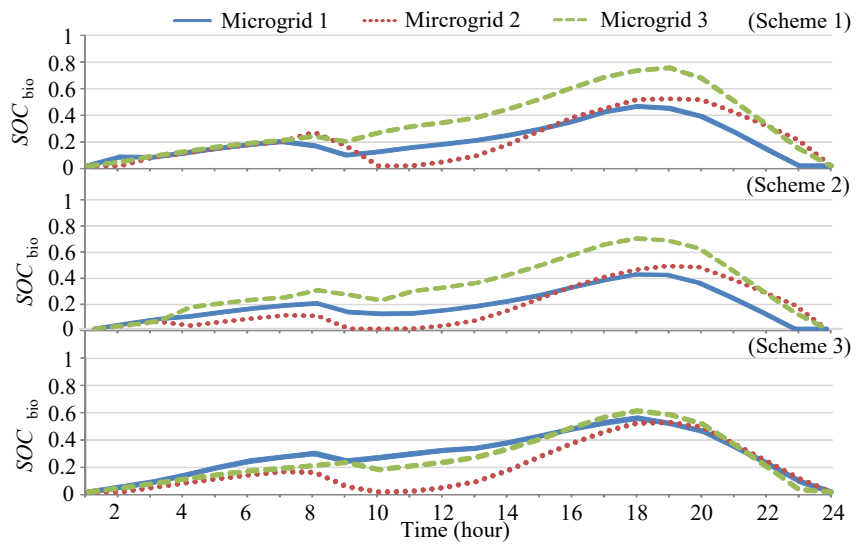

Fig. $8 \mathrm{SOC}$ of biogas storage of microgrids in schemes 1-3

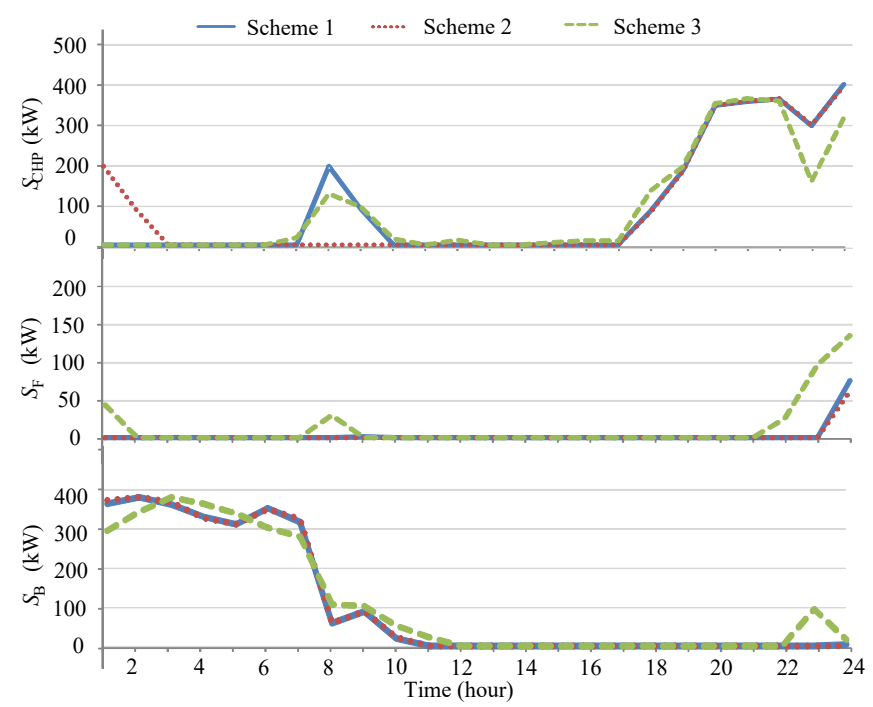

Fig. 9 Daily outputs of CHP, furnace, and boiler in schemes 1-3

Table I gives the quantitatively comparisons with schemes with and without multi-resource trading over operating costs and their corresponding payments. It can be found that, with the multi-resource trading, the system operating cost of the interconnected microgrids is decreased by up to $18.83 \%$ from 1343.48 to 1090.50 . More specifically, resource trading decreases the operating costs of microgrids 1 and 3 , which is contrary to microgrid 2 . It is because that profit-seeking microgrid 2 delivers more power and biogas to microgrids 1 and 3 instead of feeding back to the electricity market, and microgrid 2 receives 131.81 from microgrid 3 . It also can be found that every microgrid benefits through resource trading taking into account the comprehensive influences of costs and payments. Compared with scheme without resource trading, the individual costs of microgrids are reduced by $31.08 \%$, 
$21.65 \%$, and $12.35 \%$, respectively. This verifies the effectiveness and superiority of the proposed multi-energy and communication trading scheme, which encourages microgrids to share their multiple types of geo-distributed resources.

TABLE I

COMPARATIVE PERFORMANCE RESUltS OF SCHEMES 1 AND 3

\begin{tabular}{ccccc}
\hline \hline Microgrid & 1 & 2 & 3 & Total \\
\hline Cost (no trading) $(\$)$ & 271.35 & 389.38 & 682.75 & 1343.48 \\
Cost (with trading) $(\$)$ & 206.56 & 436.87 & 447.07 & 1090.50 \\
Payment (for trading) $(\$)$ & -19.54 & -131.81 & 151.35 & 0 \\
Cost+Payment (with trading) $(\$)$ & $\mathbf{1 8 7 . 0 2}$ & $\mathbf{3 0 5 . 0 6}$ & $\mathbf{5 9 8 . 4 2}$ & $\mathbf{1 0 9 0 . 5 0}$ \\
\hline \hline
\end{tabular}

Table II provides the quantitatively comparisons of schemes 1-3 over operating cost, biogas yield, battery degradation cost, electricity procurement, and max-average utilization rate. Noted that max-average utilization rate of cloudlet is calculated as the maximum of average utilization rate of individual microgrid cloudlet. It can be observed that, compared with schemes 2 and 3, scheme 1 can better quality of energy and communication services with lower electricity procurement and resource utilization. Compared to the scheme 2, the system operating cost of scheme 1 is reduced by $1.55 \%$ and the communication resource utilization level is reduced by $45.45 \%$. As for the scheme 3, the system operating cost and communication resource utilization level of scheme 1 are decreased by $18.83 \%$ and $47.83 \%$, respectively.

It is also found that the proposed methodology can offer diversified renewable energy utilization approaches through the synergies among multi-energy conversion and storage. As a consequence of the $\mathrm{P} 2 \mathrm{P}$ energy trading, microgrids in scheme 1 and 2 prefer to share their available storage and demand response resources for renewable utilization maximization, thereby reducing the degradation cost and discomfort cost in Table II. In summary, case studies can confirm the superiority of the proposed scheme on cost-efficient multi-microgrid multi-energy and communication management, especially on the enhancements on operational economy and resource utilization.

TABLE II

Comparative Performance Results of SCHEMES 1-3

\begin{tabular}{cccc}
\hline \hline Scheme & 1 & 2 & 3 \\
\hline System operating cost $(\$)$ & $\mathbf{1 0 9 0 . 5 0}$ & 1107.64 & 1343.48 \\
Battery degradation cost $(\$)$ & $\mathbf{1 9 . 9 2}$ & 19.94 & 20.17 \\
Discomfort cost $(\$)$ & 103.84 & $\mathbf{1 0 1 . 5 4}$ & 141.87 \\
Electricity procurement $(\mathrm{kWh})$ & $\mathbf{4 3 4 7 . 4 5}$ & 4446.96 & 4671.51 \\
Max-average utilization $(\mathrm{pu})$ & $\mathbf{0 . 3 6}$ & 0.66 & 0.69 \\
\hline \hline
\end{tabular}

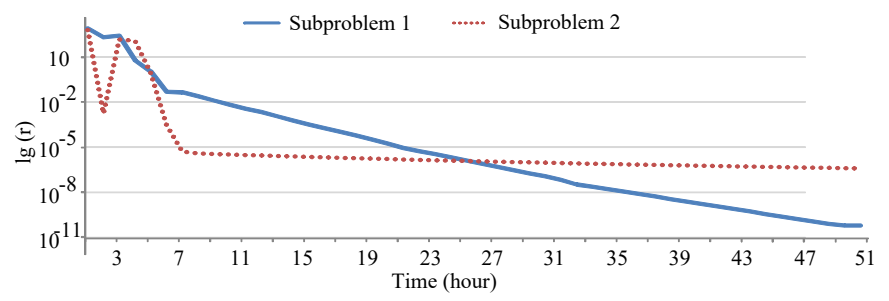

Fig. 10. Convergence process of subproblem 1 and 2

Fig. 10 shows the convergence process of max-residual errors of subproblems (12) and (13). It can be found that, with the implementation of the proposed distributed algorithms, the $\mathrm{P} 2 \mathrm{P}$ resource trading problem can be efficiently solved with the standard solver, and the gaps between centralized and distributed approaches are less than $0.5 \%$. The resulting statistics thus showed that, although they aren't strictly optimal values, the satisfactory upper bounds to the centralized optimal values can be obtained with the proposed distributed approach with better trading information privacy and decision-making independence.

\section{CONCLUSIONS}

This paper proposes a distributed transactive multi-resource trading framework for the optimal synergies of heterogeneous microgrids, and a bargaining-based multi-lateral mechanism is designed to encourage proactive resource trading among interconnected microgrids, such that diverse multi-energy generation/load profiles are leveraged to bring mutual benefits. The multi-microgrid multi-energy and communication trading problem is decomposed into social multi-resource allocation subproblem and payoff allocation subproblem, which are further solved and decentralized as multiple microgrid-level decision-makings based on fully-distributed ADMM approaches. It can be found from case studies that, as a consequence of the proactively trading of multi-energy and communication resources, the proposed scheme can outperform others on system operational economy and resource utilization with satisfactory trading payoff sharing. Furthermore, the proposed approach is fully-distributed with only necessary trading information shared, thereby preserving the privacy and resource-autonomy.

It still should be noted that the initial focus of this work is to model the multi-microgrid multi-resource trading problem with perfect communication assumed under a quasi-static environment. More investigations can be further devoted to understand the impact of imperfect communication (e.g., delay and packet loss), which is an important and main part of our future works.

\section{ACKNOWLEDGEMENTS}

The authors gratefully acknowledge the support of the State Key Laboratory of Alternate Electrical Power System with Renewable Energy Sources, Beijing, China.

\section{REFERENCES}

[1] B. Zhou, D. Xu, C. B. Li, et al., "Optimal scheduling of biogas-solar-wind renewable portfolio for multi-carrier energy supplies," IEEE Trans. Power Syst., vol. 33, no. 6, pp. 6229-6239, Nov. 2018.

[2] M. N. Alam, S. Chakrabarti and A. Ghosh, "Networked microgrids: state-of-the-art and future perspectives," IEEE Trans. Ind. Informat., vol. 15, no. 3, pp. 1238-1250, March 2019.

[3] D. Xu, B. Zhou, Q. W. Wu, C. Y. Chung, C. B. Li, S. Huang, and S. Chen, "Integrated modelling and enhanced utilization of power-to-ammonia for high renewable penetrated multi-energy systems," IEEE Trans. Power Syst., DOI: 10.1109/TPWRS.2020.2989533.

[4] K. Anoh, S. Maharjan, A. Ikpehai, et al., "Energy peer-to-peer trading in virtual microgrids in smart grids: a game-theoretic approach," IEEE Trans. Smart Grid, DOI: 10.1109/TSG.2019.2934830, in press, 2019.

[5] A. Paudel, K. Chaudhari, C. Long and H. B. Gooi, "Peer-to-peer energy trading in a prosumer-based community microgrid: a game-theoretic model," IEEE Trans. Ind. Electron., vol. 66, no. 8, pp. 6087-6097, Aug. 2019.

[6] G. E. Asimakopoulou, A. L. Dimeas, and N. D. Hatziargyriou, "Leader-follower strategies for energy management of multi-microgrids," IEEE Trans. Smart Grid, vol. 4, no. 4, pp. 1909-1916, Dec. 2013.

[7] H. Nunna and S. Doolla, "Multiagent-based distributed-energy-resource management for intelligent microgrids," IEEE Trans. Ind. Electron., vol. 60, no. 4, pp. 1678-1687, Apr. 2013.

[8] M. H. Cintuglu and O. A. Mohammed, "Behavior modeling and auction architecture of networked microgrids for frequency support," IEEE Trans. Ind. Informat., vol. 13, no. 4, pp. 1772-1782, Aug. 2017. 
[9] W. Liu, D. Qi and F. Wen, "Intraday residential demand response scheme based on peer-to-peer energy trading," IEEE Trans. Ind. Informat., vol. 16, no. 3, pp. 1823-1835, Mar. 2020.

[10]H. Kim and M. Thottan, "A two-stage market model for microgrid power transactions via aggregators," Bell Labs Tech. J., vol. 16, no. 3, pp. 101107, Dec. 2011.

[11]C. Li, Y. Xu, X. Yu, C. Ryan, and T. Huang, "Risk-averse energy trading in multi-energy microgrids: a two-stage stochastic game approach," IEEE Trans. Ind. Informat., vol. 13, no. 5, pp. 2620-2630, Oct. 2017.

[12]W. Pei, Y. Du, W. Deng, et al., "Optimal bidding strategy and intra-market mechanism of microgrid aggregator in real-time balancing market," IEEE Trans. Ind. Informat., vol. 12, no. 2, pp. 587-596, Apr. 2016.

[13]A. M. Jadhav, N. R. Patne and J. M. Guerrero, "A novel approach to neighborhood fair energy trading in a distribution network of multiple microgrid clusters," IEEE Trans. Ind. Electron.,vol. 66,no. 2,pp.1520-1531,Feb. 2019.

[14]N. Liu, X. Yu, C. Wang and J. Wang, "Energy sharing management for microgrids with PV prosumers: a Stackelberg game approach," IEEE Trans. Ind. Informat., vol. 13, no. 3, pp. 1088-1098, June 2017.

[15] M. Hu, Y. Wang, X. Lin and Y. Shi, "A decentralized periodic energy trading framework for pelagic islanded microgrids," IEEE Trans. Ind. Electron., DOI: 10.1109/TIE.2019.2942551, in press, 2020.

[16] T. Morstyn, N. Farrell, S. Darby, and M. Mcculloch, "Using peer-to-peer energy-trading platforms to incentivize prosumers to form federated power plants," Nat. Energy, vol. 3, pp. 94-101, Feb. 2018.

[17] Y. Xu, H. Sun and W. Gu, "A novel discounted min-consensus algorithm for optimal electrical power trading in grid-connected DC microgrids," IEEE Trans. Ind. Electron., vol. 66, no. 11, pp. 8474-8484, Nov. 2019.

[18]A. Paudel, M. Khorasany and H. B. Gooi, "Decentralized local energy trading in microgrids with voltage management," IEEE Trans. Ind. Informat., DOI: 10.1109/TII.2020.2980160.

[19] M. Khorasany, Y. Mishra and G. Ledwich, "A decentralized bilateral energy trading system for peer-to-peer electricity markets," IEEE Trans. Ind. Electron., vol. 67, no. 6, pp. 4646-4657, Jun. 2020

[20]N. Liu, J. Wang, and L. Wang, "Hybrid energy sharing for multiple microgrids in an integrated heat-electricity energy system," IEEE Trans. Sustain. Energy, vol. 10, no. 3, pp. 1139-1151, Jul. 2019.

[21]D. Xu, B. Zhou, K. W. Chan, et al. "Distributed multi-energy coordination of multi-microgrids with biogas-solar-wind renewables," IEEE Trans. Ind. Informat., vol. 15, no. 6, pp. 3254-3266, Jun. 2019.

[22]Z. Li, J. Kang, R. Yu, D. Ye, Q. Deng and Y. Zhang, "Consortium blockchain for secure energy trading in industrial internet of things," IEEE Trans. Ind. Informat., vol. 14, no. 8, pp. 3690-3700, Aug. 2018.

[23] W. Hou, L. Guo and Z. Ning, "Local electricity storage for blockchain-based energy trading in industrial internet of things" IEEE Trans. Ind. Informat., vol. 15, no. 6, pp. 3610-3619, Jun. 2019.

[24]H. Wang and J. Huang, "Incentivizing energy trading for interconnected microgrids," IEEE Trans. Smart Grid, vol.9, no. 4, pp. 2647-2657, Jul. 2018.

[25]H. Kim, J. Lee, S. Bahrami and V. Wong, "Direct energy trading of microgrids in distribution energy market," IEEE Trans. Power Syst., vol. 35, no. 1, pp. 639-651, Jan. 2020.

[26] Y. Z. Li, T. Y. Zhao, and P. Wang, et al, "Optimal operation of multi-microgrids via cooperative energy and reserve scheduling," IEEE Trans. Ind. Informat., DOI: 10.1109/TII.2018.2792441, in press, 2018.

[27] M. Latifi, A. Rastegarnia, A. Khalili, W. M. Bazzi and S. Sanei, "A self-governed online energy management and trading for smart micro/nano-grids," IEEE Trans. Ind. Electron., DOI: 10.1109/TIE.2019.2945280

[28] W. Liu, W. Gu, J. Wang, W. Yu and X. Xi, "Game theoretic non-cooperative distributed coordination control for multi-microgrids," IEEE Trans. Smart Grid, vol. 9, no. 6, pp. 6986-6997, Nov. 2018.

[29]R. Yu, J. Ding, S. Maharjan, et al., "Decentralized and optimal resource cooperation in geo-distributed mobile cloud computing," IEEE Trans. Emerging Topics in Computing, vol. 6, no. 1, pp. 72-84, Jan.-March 2018.

[30]E. Ahvar, A. Orgerie and A. Lébre, "Estimating energy consumption of cloud, fog and edge computing infrastructures," IEEE Trans. Sustain. Computing, DOI: 10.1109/TSUSC.2019.2905900, in press, 2019.

[31] C. Wang, T. Zhang, F. Luo, F. Li, and Y. Liu, "Impacts of cyber system on microgrid operational reliability," IEEE Trans. Smart Grid, vol. 10, no. 1, pp. 105-115, Jan. 2019.

[32]H. Wang, J. Huang, X. Lin, and A. H. Mohsenian-Rad, "Proactive demand response for data centers: A win-win solution," IEEE Trans. Smart Grid, vol. 7, no. 3, pp. 1584-1596, May 2015.

[33]D. Jin, Z. Li, C. Hannon, et al., "Toward a cyber resilient and secure microgrid using software-defined networking," IEEE Trans. Smart Grid, vol. 8, no. 5, pp. 2494-2504, Sep. 2017.
[34] R. Yu, J. Ding, X. Huang, et al., "Optimal resource sharing in 5G-enabled vehicular networks: a matrix game approach," IEEE Trans. Vehicular Technology, vol. 65, no. 10, pp. 7844-7856, Oct. 2016.

[35] J. Zhao, H. Wang, Y. Liu, et al., "Coordinated restoration of transmission and distribution system using decentralized scheme," IEEE Trans. Power Syst., vol. 34, no. 5, pp. 3428-3442, Sep. 2019.

[36]D. Xu, Q. Wu, B. Zhou, et al. "Distributed multi-energy operation of coupled electricity, heating and natural gas networks," IEEE Trans. Sustain. Energy, DOI: 10.1109/TSTE.2019.2961432, in press, 2020.

[37]J. Li, C. Zhang, Z. Xu, J. Wang, J. Zhao, and Y.J. Zhang, "Distributed transactive energy trading framework in distribution networks," IEEE Trans. Power Syst., vol. 33, no. 6, pp. 7215-7227, Nov. 2018.

[38]H. Yang, S. Li, Q. Li, and W. Chen, "Hierarchical distributed control for decentralized battery energy storage system based on consensus algorithm with pinning node," Protection Control Modern Power Syst., vol. 3, no. 6, pp. 1-9, Feb. 2018, DOI:10.1186/s41601-018-0081-5.

[39] X. Lin and T. M. Lok, "Distributed power control for one-to-many transmissions in gaussian interference channels," IEEE Trans. Communications, vol. 60, no. 8, pp. 2363-2375, August 2012.

[40] W. J. Mai and C. Y. Chung, "Economic MPC of aggregating commercial buildings for providing flexible power reserve," IEEE Trans. Power Syst., vol. 30 , no. 5 , pp. 2685-2694, Sep. 2015.

[41] W. Thomson, "Cooperative models of bargaining," Handbook of game theory with economic applications, vol. 2, pp. 1237-1284, 1994.

[42] J. F. Nash, "The bargaining problem," Econometrica: J. Econometric Soc., vol. 18, no. 2, Apr. 1950.

[43] S. Boyd, N. Parikh, E. Chu, et al., "Distributed optimization and statistical learning via the alternating direction method of multipliers," Found. Trends Mach. Learn., vol. 3, no. 1, pp. 1-122, Jul. 2011.

[44] W. Huang, N. Zhang, C. Kang, et al, "From demand response to integrated demand response: review and prospect of research and application," Protection Control Modern Power Syst., vol. 4, no. 12, pp. 1-13, May. 2019, DOI:10.1186/s41601-019-0126-4.

[45] GAMS [Online]. Available: http://www.gams.com/. 